\title{
Interactive Collaboration and Foreign Language Teaching Methodology
}

\author{
Miao Wang \\ Nan Yang Institute of Technology, Nanyang 473004, China \\ wmaugust1982@163.com
}

Keywords: Interactive Collaboration; foreign language teaching; foreign language teaching.

\begin{abstract}
Nowadays, A good and effective interaction and cooperation during the students and teachers during the foreign language learning is the vital standard to weigh the quality of the foreign language learning and teaching. This paper is mainly talking about the theory of collaborative concept as well as the important role of the interactive collaboration in the foreign language teaching and how to use the principle of the interaction and the collaboration to guide the foreign language teaching in order to improve the effects of foreign language leaning.
\end{abstract}

\section{Introduction}

Now, in China, interactive, cooperative teaching is the most popular foreign language learning method in universities, colleges and high schools. Interactive teaching approach indicated to a larger extent that teachers should go beyond language structures in order to improve the learners' communicative competence through interaction. Students interaction can be interpreted as the interaction and influence in various forms, such as nature and levels among students in a certain background and specific situation of classroom culture. In the English learning classes, interaction and cooperation between the students and teachers are regarded as the efficient way to master the language. Group cooperation are received the warm welcome among students. The paper explores the theoretical foundation in the following paragraph.

\section{Theory}

The most natural and fundamental way for human beings to use language is dialogue (Pickering and Garrod 2004), The mechanism of using language is naturally latent in the conversation. The purpose of learning language is to use it. It is definitely beneficial to foreign language teaching and the foreign language learning after they know the purpose of learning language. Dialogue is a kind of human social interaction. Interaction needs collaboration between the people, collaboration needs alignment which is the important method in the conversational interaction. Based on Interactive Collaborative Model of psycho-linguistic research, during the conversation, the speakers will form a dynamic concept or situation model about the listeners' space, intention, context (Zwaan and Radvansky). The two sides coordinate with each other, stimulate each other and ideas call forth ideas, establish mutual convergence while the speaker and the listener interact between each other in the course of communication, so that the listener can understand the meanings of words which is considered to be an alignment. will promote the understanding, ensure the smooth conversation. Generally speaking, from the view of the social cognition, alignment is thought to be a complicated process in which the human mind and body interact and coordinate the society. Alignment is the key factor to acquire the second foreign language.

\section{Application}

\subsection{Alignment and Interaction Combined Together}

Alignment will reflect in the interaction (Pickering and Garrod2004). If A used the word "chef" in the conversation, the B will also use "chef" instead of "cook", so the conversation is the most efficient interaction way of alignment. In the dialogue, there are not only listening activity but also the the 
speaking activity. Listening and speaking coordinate each other and influence each other which is defined as two-way collaboration. Two-way collaboration greatly improves the synergistic effect of language. As a result, it is easy to know why the child can learn the native language very well in a short time. In the process of interaction and synergy, the parents will coordinate the rate of speech, tone, diction and the sentences according to the Children's ability to understand the world and their communicative competence.

Earlier research by Long (1983) found that second language learners have improved a lot when interaction has been done with the people who are above their foreign language proficiency, especially the interaction with the native speakers. Therefore, he put forward the Interaction Hypothesis. The mechanism of interaction is Synergy, alignment is study. No alignment, no progress.

\subsection{The Content Decides the Alignment}

Alignment is significantly associated with the appeal of content. The language could not exist without the contents. During the conversation, an interesting topic will attract the listeners' attention, so the listener would like to take part in the topic, the fantastic content will inspire the imagination and resonance.

\subsection{The Beliefs and Emotions Reflects the Synergy}

Synergy also has to do with people' beliefs and emotions. Liking or not also belong to collaboration. Emotion also belongs to collaboration which is very important for learning a foreign language. For example, A teacher's personality and knowledge are appreciated by the students, and they will get the students' emotional approval or cooperation and can easily arose the students' learning enthusiasm in their lessons. Besides. Students like listening to his lectures, the contents of the teaching were easily absorbed by the students. Once the students are disgusted with the class, and the emotional cooperation chain between teachers and students have broken, the effects of learning will become bad. Recent research abroad has found the most important factor influencing foreign language learning is whether students like teachers or not in class.

\subsection{Collaboration Decides Learning Results.}

Collaboration decides the foreign language learning results. The learner is the whole of all language learning variables and the initiator that initiates the synergy. The learning effect is produced in synergy. The synergy between emotional patterns, language structures and emotions and learning variables means that synergy is omnidirectional. Learners' wholehearted devotion to learning is equal to all-directional synergy which strengthens the synergistic effect. As far as foreign language learning is concerned, what kind of learning method produces stronger synergistic effect? Interaction with native speakers is undoubtedly the best way to learn a foreign language well. Listening and speaking dynamic interaction, the context information is very rich.The more dynamic interaction we have during listening and speaking activities, the richer context information we will get. The synergistic effect is remarkable. It can build up the language sense and acquire the idiomatic language.

\subsection{Interaction is a Two-Way Street}

Because not everyone has a chance to talk to foreigner, the need of communicating with the native speakers could not satisfy. From the perspective of improving foreign language use, reading classic foreign language novels is a good way to promote. The excellent content of the novel is rich in context and rich in language standard, which is easy to memorize and help to improve the language level. However, Synergy is a single term between the reader and the writer by means of reading activity, writing exercises also need to do after the reading the novels. As a result, in order to the shortchanges, reading and writing combine to increase interaction and enhance synergy. Writing after the reading is a useful way to improve the language. The method of writing a story based on what you read is beneficial to students' foreign learning. In the reading lesson, the teacher can pick out a story or a book with a plot or philosophy, require students to read for the previous section, and write the following plot according to your reading which helps students build contextual model to use their imagination and creativity. The way of writing after reading the novels forces learners to comprehend the story and try to coordinate, At the same time, the catalytic structure is activated in conjunction with the content. In the process of writing, students constantly review and refer to the original text, Consciously or unconsciously imitate some of these expressions to reduce the expression barrier. Just 
as speaking and listening to combine to strengthen synergies in the dialogue, writing combined reading continue to help strengthen the interaction between readers and reading, to strengthen the coordination between readers and reading the plot, strengthening project language and reading together.

\subsection{Error Correction is Not a Good Way.}

More, a noticeable phenomenon in foreign language teaching should be attached great concern. Teachers and students often hold that error corrections are the stepping stones to foreign language learning. Teachers see mistakes correction as their responsibility, and the more careful they are, the more responsible they are. The idea of interactive collaboration tells us that over-correction should not be advocated in learning. Every little progress for a foreign language learner makes depends mainly on the synergy between the learner and the correct language input rather than pragmatic failure. The correct way to learn a foreign language is to make a lot of contact with the correct language input in the appropriate context and to squeeze the wrong space. The synergistic effect can explain some foreign language teaching phenomena. The English corner for improving spoken English is often difficult to keep up. In addition to the lack of communication, one of the important reason is that the students who attend the English corner often talk with the learners below their English level, the English sense of achievement brought by the synergy effect is low, conversation power drops rapidly.

\section{Conclusion}

In a all, in recent years, collaborative research has opened a new perspective for us to know more about foreign language learning and improve foreign language teaching. Teaching and learning languages should not forget such a common sense. Cooperation is the need for human survival and development. Language is the product of human cooperation. Cooperation must be synergistic, synergy must have a learning effect, and the characteristics of cooperation must be reflected in the use of language. Based on the theory of interaction synergy, what should we adopt to enhance the cooperation and synergy in foreign language learning. Teaching and learning foreign languages should make full use of the cooperative features of language use, create collaborative conditions in learning activities, and enable students to actively participate in cooperative activities. So in the process of teaching and learning a foreign language, teachers should carefully design to ensure that the teaching content is lively and interesting in order to attract the attention of students and inspire them to join in the activity in the class. Besides, a teacher should establish a good emotional connection with the students and gain the love and respect from students. As a result, a good teacher should be patient and responsible and affectionate with the teaching. One of the basic conditions for a good foreign language teacher is to have a strong ability to use foreign languages as a collaborative model for students' language. In the class, the teacher uses the interactive way to organize the lessons, on the one hand teachers adopt the method of speaking after the listening, on the other hand require them to read excellent works, ask the students to write a composition after the reading, reading and writing are done at the same time. The students need to link what they learn with practice. Practice makes perfect. Strengthening the synergistic effect of correct language input can significantly improve learning efficiency. Therefore, in English lessons in university, colleges, high schools, even in primary school, teachers would like to utilize the interactive way and group cooperative learning to arrange the teaching and encourage the students to carry out all-round cooperation, inspire the students to read more masterpieces. The more important, the students should use English to do deeds.

\section{Acknowledgements}

This article is one of the phased results of the research on the innovation of the international talents incubation path under the "One Belt and One Road" strategy (182400410107), and the study on the development of foreign trade flow and foreign language ability of Henan Province (2017BYY012). 


\section{References}

[1]. Atkinson, D., T. Nishino. E. Churchill and H. Okada. 2007. Alignment and interaction in a sociocognitive approach to second language acquisition. the Modern Language Journal 91:169-188.

[2]. Gardner, R.1985. Social psychology and second language learning: the role of attitudes and motivation. London: Arnold

[3]. Pickering. M and s. Garrod. 2004. Toward a mechanistic psychology of dialogue. Behavioural and Brain Sciences 27:169-226 